\title{
Microbiological Approach in Diagnosing Native and Heart Valves Prosthesis Infections
}

\begin{abstract}
Background: Traditional culturing approaches either on native (NV) or on prosthetic valves (PV) are still not efficient in detecting pathogens responsible of infection. In fact, despite the continuous development of microbiological technologies, a truly valid technique that could be used as reference has yet to be found. Here we present a revised version of traditional culture methods based on a pre-treatment of both NVs and PVs by DL-dithiothreitol (DTT).
\end{abstract}

Methods and findings: A total of 79 specimens were included in the study: 54 were NVs and 25 were PVs. We compared the results of both culturing methods and molecular assays performed on NVs/PVs collected in two different periods named pre-DTT and post-DTT, respectively. The protocol consisted in treating NV/PV by an appropriate volume of DTT, following which the suspension of bacteria/DTT was used for culture and molecular assay.

In pre-DTT period five specimens were culture-positive and one was positive by molecular assay only $(1 / 20 ; 5 \%)$, showing a culture positivity rate of $25 \%(5 / 20)$. In the post-DTT period, of 59 specimens processed, 19 were culture positive (19/59; 32\%). Moreover, PCRs performed on specimens treated with DTT contributed to the identification of six additional positive specimens plus an identification of poly-microbial infection lost by culture $(7 / 59 ; 12 \%)$.

Conclusion: Our findings show that the use of DTT can be helpful in increasing the identification of microorganisms involved in NV/PV infections. Given its simple and cost-effective use and considering the issue that this technique does not require any specific instrumentation, it could easily be introduced in any laboratories. However, since our study included a limited number of specimens, more extensive studies are needed to further confirm our results.

Keywords: Heart Valve infection - Vascular prosthesis - Microbiological diagnosis • Native valves - Endocarditis

Submitted: 30 May 2017; Accepted: 19 June 2017; Published online: 21 June 2017

\section{Introduction}

Heart, blood vessels and blood are three fundamental components of circulatory system (CS), and their integrity is essential for the normal distribution of both oxygen and nutrients to anybody sites. On the other hand stenosis, aneurysm and valve defects represent common problems altering the normal functionality of CS. One important result of altered functionality of heart valves could be the infective endocarditis (IE) [1]. The latter remains a frequent disease with constantly changing features and of its clinical pictures, in part due to a more frequent use of invasive medical procedures (e.g. both intravenous and intracardiac devices implant), or potentially risky behaviours such as: intravenous drug use, body piercing and
Carla Fontana',2*, Marco Favaro', Pasquale Sordillo ${ }^{3}$, Cesare Sarrecchia ${ }^{3}$, Silvia Minelli2 ${ }^{2}$ Maria Cristina Bossa², Anna Altieri², and Cartesio Favalli',2

'Department of Experimental Medicine and Surgery, University of Rome Tor Vergata via Montpellier 100133 Rome, Italy

2Department of Microbiology and Virology lab, University of Rome Tor Vergata via Montpellier 100133 Rome, Italy

${ }^{3}$ The Clinical Infectious Diseases, University of Rome Tor Vergata via Montpellier 100133 Rome, Italy

*Author for correspondence: carla.fontana@uniroma2.it 
tattooing [1-4]. Moreover, IE is more common in men and in elderly patients and it is often associated with other diseases such as diabetes, cancer, alcoholism [1]. Both native and prosthetic valves may be involved in IE. The first event is bacterial adherence to damaged valves, the second step involves persistence and growth of bacteria within the cardiac valves lesions, usually associated with local extension and tissue damage [5]. Surgery, by replacing native valve as well as tract of vessels by prosthesis implants, generally returns the lost functionality [6]. Nevertheless, the surgical approach is burned by several risks among which infections represent a paramount aspect. The prosthesis, in fact, predisposes to device related infections, especially those caused by microorganisms, which are able to adhere to a variety of surfaces and produce biofilm [7]. Prosthetic valve endocarditis (PVE) is an important cause of morbidity and mortality, its overall incidence has been reported to be between 0.3 and $1.2 \%$ per patient/year and mortality can achieve $50 \%$ [8-10]. PVE represents $20 \%$ of all cases of IE, with an increasing incidence [4]. The timing of the infection reflects different pathogenic mechanisms and PVE are usually classified in two groups according to the time elapsed from surgery: early-onset and lateonset. There is no complete agreement regarding the cut-off time to classify a PVE. Some authors consider 1 year, others 60 days after the valve placement surgery for the late-onset and $<1$ year or $<60$ days for the early-onset PVE $[8,11]$. In any case diagnosis of PVE can be really challenging. Various criteria have been proposed during the time. In 2000, the modified Duke criteria were recommended and these were based on clinical, echocardiographic and biological findings, as well as the results of blood cultures and serology. Albeit, modified Duke's criteria show a low diagnostic accuracy in the case of PVE, the collection of blood cultures remains the gold standard approach [4]. On the other hand, significant is the proportion of PVEblood culture negative [4]. The latter was in part due to the fact that patients received antibiotics before blood was cultured [12]. On the other hand, the emergence of pathogens difficult to cultivate, of pathogens viable but not cultivable as well as microorganisms producing biofilm complicates the diagnostic scenario $[4,13]$. Even if Munoz et al. reported that heart valve culture does not have good sensitivity (7.8 to $17.6 \%)$ and may be contaminated during manipulation, thus suggesting their cultivation only in patients with a strong suspicion of IE, it is still of great importance to proceed to culture either native or prosthetic valves after their substitution [14]. Culture approach is paramount not only to define the epidemiological asset of NV/PV infections, but also in order to performing antimicrobial susceptibility testing on the isolates in favour of a more appropriate treatment. On the other hand it is equally clear that the traditional microbiological approach on native/ prosthetic valve culture is still not be able to satisfy all medical needs, so we have thought to process these specimens by coupling a revised version of traditional culture methods with molecular assays.

\section{Methods}

\section{Specimens}

Prosthetic (PVs) as well as native valves (NVs) were removed aseptically from patients with a suspicion of IE who underwent cardiac surgery and promptly sent to laboratory for microbiology workup. A total of 79 specimens (from 79 different patients) were included in the study (in the period comprises from January 2010 to March 2017): 54 were NVs and 25 were PVs. The study has been conducted by comparing the results of two different periods: i) from January 2010 to December 2012, during which NTs and PVs were processed by traditional culturing methods (named pre-DTT); ii) from January 2013 to March 2017 when NTs and PVs were cultured by a DTT-protocol (called: post-DTT).

\section{PVs/NVs Culturing}

DTT protocol consisted in treating NVs as well as PVs by an appropriate volume of DTT directly in the same sterile container used for collection in the surgery room (usually that used for urine sampling). DTT is a sulfhydryl compound (empirical formula C4H10O2S2, MW 154.2) commonly used in clinical microbiology for liquefying specimens such as sputum. DTT can reduce disulphide bounds between polysaccharides and neighbouring proteins and therefore can works against biofilm structure [15]. DTT was used with the intention of obtaining a disorganization of biofilm adherent to PV/NV and gaining planktonic cells that could be more easily cultivated. DTT was used at the working solution contains $0.1 \% \mathrm{w} / \mathrm{v}$ of reagent. The volume of DTT added depends on the characteristics alongside the dimension of PV/NV delivered to the laboratory, though a good approach is to use $1-2 \mathrm{~mL}$ of DTT for each $\mathrm{cm}^{2}$ of PV. DTT-treated PV/NV were maintained at room temperature in slow agitation (on an automatic rotor at about $100 \mathrm{rpm}$ by orbital oscillation) for $15 \mathrm{~min}$. Afterwards, the suspension of bacteria/DTT was transferred in sterile tube and centrifuged at $3000 \mathrm{~g} \times 10 \mathrm{~min}$., the supernatant were removed and the pellet was suspended in $1 \mathrm{~mL}$ of sterile saline solution ( $0.45 \%$ Sodium Chloride) (PESL). $0.5 \mathrm{~mL}$ of PESL were enriched by culturing 
in a liquid media using HB\&L Culture Kit vial (Alifax, Padova Italy) also supplemented with HB\&L DEB Kit (Alifax) in order to guarantee the growth of the fastidious microorganism. Vials were incubated on SIDECAR automated system (Alifax) for $6 \mathrm{~h}$. The SIDECAR is the automated version of HB\&L together with the ALFRED system and it is also based on laser light scattering nephelometer technology [16,17]. At the end of the instrumental protocol, specimens were seeded onto a set of different media (all provided by bioMeriux; Marcy l'Etoile. France), namely: Columbia CNA (two plates in order to perform two different incubation: in ordinary atmosphere and in anaerobic condition), Mac Conkey agar, Saboraud Destrose Agar; Chocolate agar and Schaedler agar (the latter incubated in anaerobic condition). Plates were maintained in observation for 5 days. Microorganisms' growth from positive specimens was identified using MALDI TOF MS technology (Bruker Daltonics GmbH, Bremen, Germany) while the antimicrobial susceptibility testing was performed using Vitek 2 system (bioMerieux) [18]. For each patient, culture results of PV/NV were also matched with those of blood cultures (BC) collected in the days before the cardiac surgery. For this purpose blood from each patient was collected in at least three sets of BD Bactec Plus Aerobic/F (Becton Dickinson, $\mathrm{NJ}$, USA). BCs were maintained in continuous monitor incubation in the Bactec FX systems (Becton Dickinson), concluded negative after 5 days or otherwise promptly removed and processed when flagged positive by instrument. Positive BCs were seeded on a set of three plates of Chocholate agar, incubated at $35 \pm 2^{\circ} \mathrm{C}$ under aerobic, micro aerobic and anaerobic condition, respectively. Isolates from positive BCs were identified as above reported.

\section{Molecular assay on PVs/NVs}

Culture negative $\mathrm{PV} / \mathrm{NVs}$ were also tested by molecular assay as recommended by Habib et al. [4]. Particularly, the remaining $0.5 \mathrm{~mL}$ of PESL, were extracted in order to obtain nucleic acid using the EZ1 Robot (Qiagen Inc., Valencia, CA) and $1 \mu \mathrm{l}$ of the eluate was used for both bacterial and fungal broad-range PCRs, the first by amplifying $16 \mathrm{~S}$ rDNA gene (primers $8 \mathrm{~F}$ and $516 \mathrm{R}$ ) and the second by amplifying ITS region (primer 18SF, and 28SR). Three microliters of the amplicons obtained were then used for the sequencing reaction using the BigDye Terminator v1.1 Cycle Sequencing-ready reaction kit (ABI PRISM) (Applera, Foster City, CA, USA). The electropherograms were run on the ABI 310 and genetically analysed on NCBI. The electropherograms would have been analysed by Mixed Rip Seq (Isentio, Bergen, Norway), in the event of mixed sequences (as it was in polymicrobial infections) [19].

\section{Results}

In order to verify if the DTT protocol was more efficient in recovering live bacteria than the traditional culture method, we compared the results of culturing on both NVs and PVs, before and after the introduction of the DTT-protocol (named pre-DTT and post-DTT, respectively). It was, in fact, not feasible to divide NVs or PVs in two different portions to destine to the two different protocols without increasing the risk of a possible contamination or by altering culture results due to the considerable reduction of the size of the fragment sent to the culture. For these reasons, we chose to compare samples of two different periods: before and after the introduction of DTT-protocol. A total of $54 \mathrm{NVs}$ and $25 \mathrm{PVs}$ were examined. A total of 20 specimens were collected and processed in the preDTT- while 59 in post-DTT-period.

NVs were: 33 native aortic valves (NAV), 18 native mitral valves (NMV) and three tricuspids (TV), respectively; while among 25 PVs examined, 20 were prosthetic aortic valves (PAV) and 5 were prosthetic mitral valves (PMV) (Table 1).

In pre-DTT period five specimens $(5 / 20)$ were culture-positive and one was positive by molecular assay only $(1 / 20 ; 5 \%)$, showing a culture positivity rate of $25 \%(5 / 20)$. While, in the post-DTT period, on 59 specimens processed, 19 were culture positive (19/59; $32 \%)$. Moreover, PCRs performed on specimens treated with DTT contributed to the identification of six additional positive specimens plus an integrated identification of mixed pathogens lost by culture (7/59; 12\%) (Table 1). Hence, either the identifications obtained by culture methods or those obtained by molecular assay have been increased by the introduction of DTT-treatment. In addition, during the post-DTTperiod, cultures allowed the identification of four polymicrobial infections (Table 1). Considering the results of blood cultures, 37 were concordantly negative with NV/PV cultures. In none cases the microorganism identified in $\mathrm{BC}$ matched with that isolated by culturing NP/PV specimens (Table 1).

\section{Discussion}

Infective endocarditis, although uncommon (two to six episodes per 100,000 habitants/year), is a severe infection hampered by a mortality ranging from 15 to $30 \%[4,20]$. Several study report different incidence of IE, but in general the scientific community agreed on the 


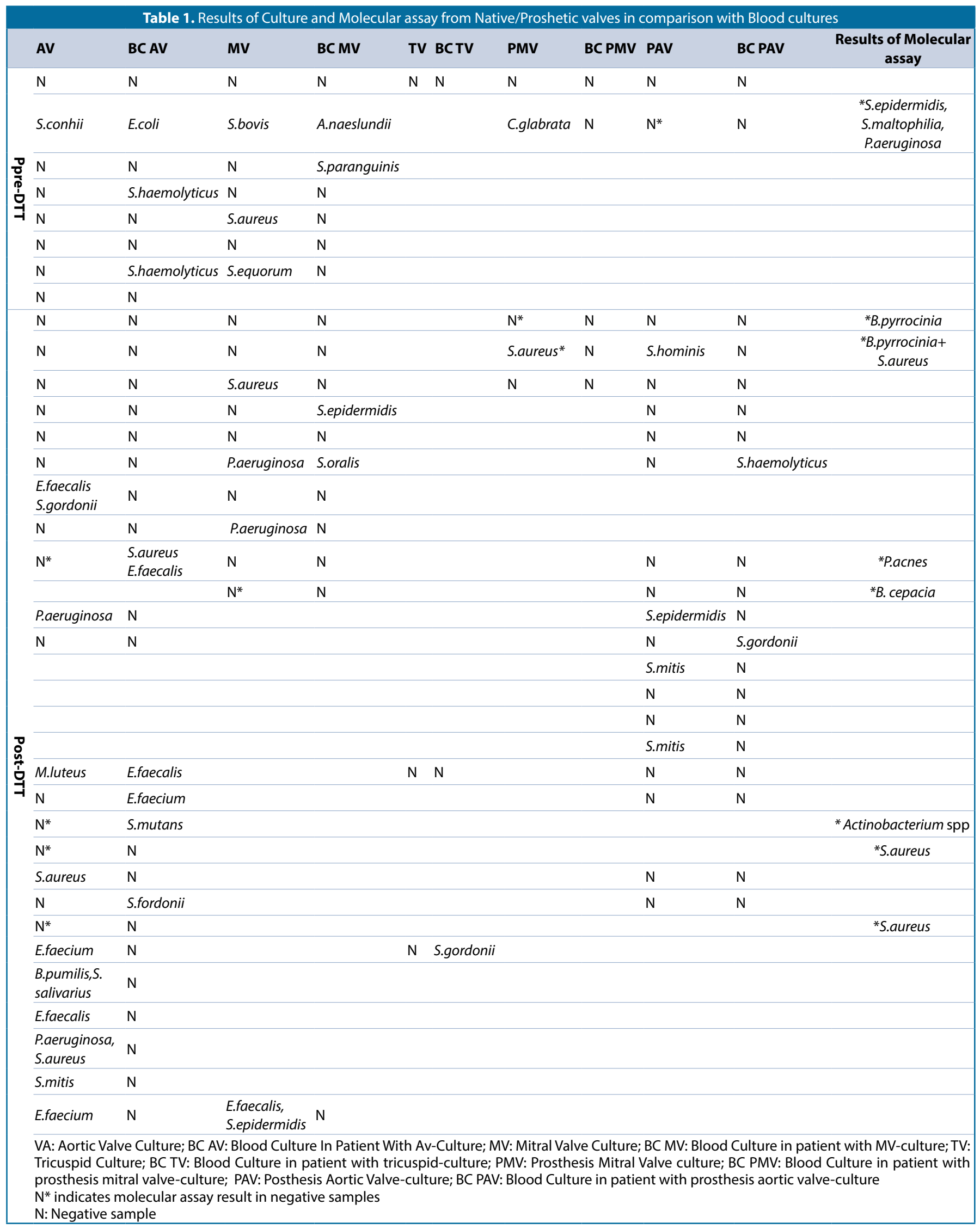


increased proportion of this syndrome [6]. The proper management of IE can be optimized if the etiological diagnosis is made [21]. Even if the diagnosis of IE is based on a strict adherence of the criteria stated in 2015 by a guideline of the European Society of Cardiology, it still remains challenging [4]. In most cases either in native or prosthetic valve infections microbiology plays a crucial role. Nevertheless, diagnosis of NVs as well as PVs infections remains a concern for microbiology laboratories, in fact, despite the continuous development of microbiological technologies, a truly valid technique that could be used as reference has yet to be found. Blood culture is still considered the cornerstone, particularly because it represents the sole procedure able to provide living microorganisms by subjecting to antimicrobial susceptibility testing (needful to set the therapy). However, on the other hand IEs with a negative blood cultures can occur in up to $31 \%$ of all cases and pose reasonable diagnostic and therapeutic dilemma [22,23]. It is also controversial the approach to systematically undergo all surgically removed NV/ PVs to microbiology observation. In fact, although a vegetation-positive culture is a major diagnostic criterion for IE, the risk of obtaining, even just from the culture, a growth of contaminant microorganisms is high [14,24-26]. Anyway, it is also well known that the sensitivity of culturing methods is low and conversely the number of endocarditis orphan of causative agent still too high. In order to respond to the latter unmet medical need, we have applied a procedure (based on the use of DTT), born to be applied to osteo-articular prosthesis, together with NVs and PVs. Our findings show that the post-DTT treatments increased the sensibility of culturing of about $7 \%$ (32 vs. $25 \%$ ) and, particularly, allowed the identification of some poly- microbial infections. Intriguing was the added value of the molecular assay, whose results have more than doubled those obtained in the pre-DTT-period (12\% vs. $5 \%$ ). On the latter aspect we can speculate that the efficacy of DTT in freeing bacteria from the biofilm could have a positive effect on the ability of molecular assay to detect a greater number of pathogens. The results of blood cultures during the two different periods did not show any significant differences between prel post DTT period, however this was in part expected because the DTT protocol does not affect or modifies in anyway the blood culture protocol, and the trends observed confirm the limit of this tool in diagnosing endocarditis $[22,23]$. We recognize that a potential bias of our study consists in the fact that we did not process each sample with both procedures (such of pre-DTT and that of post-DTT time), e.g. by dividing each removed device in two portions. Nevertheless, that was a demanded choice in order to avoid an increasing risk of contamination or worse to prejudice culturing results.

In conclusion, albeit more extensive studies shall be auspicated, particularly in order to increase the number of specimens processed by DTT, our findings show that the use of DTT can, indeed, be helpful in increasing the positivity rate of culture methods. Moreover, due to its simple and cost-effective use and particularly considering the fact that this technique does not require specific instrumentation, it can be easily introduced in any laboratories.

\section{Funding}

No external funding was received

\section{Transparency Declaration}

The authors have no conflicts of interest to declare

\section{Executive summary}

Background: Traditional culturing approaches either on native (NV) or on prosthetic valves (PV) are still not efficient in detecting pathogens responsible of infection. In fact, despite the continuous development of microbiological technologies, a truly valid technique that could be used as reference has yet to be found. Here we present a revised version of traditional culture methods based on a pre-treatment of both NVs and PVs by DL-dithiothreitol (DTT).

Methods and findings: A total of 79 specimens were included in the study: 54 were NVs and 25 were PVs. We compared the results of both culturing methods and molecular assays performed on NVs/PVs collected in two different periods named pre-DTT and post-DTT, respectively. The protocol consisted in treating NV/PV by an appropriate volume of DTT, following which the suspension of bacteria/DTT was used for culture and molecular assay.

In pre-DTT period five specimens were culture-positive and one was positive by molecular assay only (1/20; $5 \%)$, showing a culture positivity rate of $25 \%(5 / 20)$. In the post-DTT period, of 59 specimens processed, 19 were culture positive (19/59; 32\%). Moreover, PCRs performed on specimens treated with DTT contributed to the identification of six additional positive specimens plus an identification of poly-microbial infection lost by culture $(7 / 59 ; 12 \%)$.

Conclusion: Our findings show that the use of DTT can be helpful in increasing the identification of microorganisms involved in NV/PV infections. Given its simple and cost-effective use and considering the issue that this technique does not require any specific instrumentation, it could easily be introduced in any laboratories. However, since our study included a limited number of specimens, more extensive studies are needed to further confirm our results. 


\section{References}

1. Cahill TJ, Prendergast BD. Infective endocarditis. Lancet 387, 882-93 (2015).

2. Mouly S, Ruimy R, Launay O, et al. The changing clinical aspects of infective endocarditis: descriptive review of 90 episodes in a French teaching hospital and risk factors for death. J. Infect. 45, 246-56 (2002).

3. Slipczuk L, Codolosa JN, Davila CD, et al. Infective Endocarditis Epidemiology Over Five Decades: A Systematic Review. PLoS One 8(12), e82665 (2013).

4. Habib G, Lancellotti P, Antunes MJ, et al. 2015 ESC Guidelines for the management of infective endocarditis: The Task Force for the Management of Infective Endocarditis of the European Society of Cardiology (ESC). Endorsed by: European Association for Cardio-Thoracic Surgery (EACTS), the European Association of Nuclear Medicine (EANM). Eur. Heart. J. 36, 3075-128 (2015).

5. Selton-Suty C, Coco-Lecomppte T, Bernard Y, et al. Clinical and microbiologic features of multivalvular endocarditis. Curr. Infect. Dis. Rep. 12, 237-43 (2010).

6. Pant S, Patel NJ, Deshmukh A, et al. Trends in infective endocarditis incidence, microbiology, and valve replacement in the United States from 2000 to 2011. J. Am. Coll. Cardiol. 19, 2070-6 (2015).

7. Piper C, Körfer C, Horstkotte D. Prosthetic valve endocarditis. Heart 85, 590-3 (2001).

8. Lopez J, Revilla A, Vilacosta I, et al. Definition, clinical profile, microbiological spectrum, and prognostic factors of early-onset prosthetic valve endocarditis. Eur. Heart. Journal. 28, 760-5 (2007).

9. Nagpal A, Sohail MR, Steckelberg JM. Prosthetic valve endocarditis: state of the heart. Clin. Invest. 2, 803-17 (2012).

10. Hoen B, Duval X. Infective Endocarditis. N. Engl. J. Med. 368, 1425-33 (2013).

11. Baddour LM, Wilson WR, Bayer AS, et al. American Heart Association Committee on Rheumatic Fever, Endocarditis, and Kawasaki Disease of the Council on Cardiovascular Disease in the Young, Council on Clinical Cardiology, Council on Cardiovascular Surgery and Anesthesia, and Stroke Council Infective Endocarditis in Adults: Diagnosis, Antimicrobial Therapy, and Management of Complications: A Scientific Statement for Healthcare Professionals From the American Heart Association. Circulation . Circulation 15, 1435-86 (2015).

12. Lamas CC, Eykyn SJ. Blood culture negative endocarditis: analysis of 63 cases presenting over 25 years. Heart 89, 258-62 (2003).

13. Lamas CC, Fournier PE, Zappa M, et al. Diagnosis of blood culture-negative endocarditis and clinical comparison between blood culture-negative and blood culture-positive cases. Infec. 44, 459-66 (2016).

14. Munoz P, Bouza E, Marın M, et al. On behalf of the Group for the Management of Infective Endocarditis of the Gregorio Maranon Hospital. Heart Valves Should Not Be Routinely Cultured. J. Clin. Microbiol. 45, 2897-2901 (2008).

15. Drago L, Signori V, De Vecchi E, et al. Use of dithiothreitol to improve the diagnosis of prosthetic joint infections. J. Orthop. Res. 31,1694-9 (2013).

16. Fontana C, Favaro M, Minelli S, Bossa MC, Altieri A, Favalli C. A novel culturing system for fluid samples. Med. Sci. Monit.15, 55-60 (2009).

17. Fontana C, Favaro M, Bossa MC, et al. Improved diagnosis of central venous catheter-related bloodstream infections using the HB\&L UROQUATTRO ${ }^{\mathrm{rm}}$ system. Eur. J. Clin. Microbiol. Infect. Dis. 31, 3139-44 (2012).

18. Singhal N, Kumar M, Kanaujia PK, Virdi JS. MALDI-TOF mass spectrometry: an emerging technology for microbial identification and diagnosis. Front. Microbiol. 6, 791 (2015).

19. Fontana C, Favaro M, Favalli C. How Liquid Based Microbiology Can Change the Workflow in the Microbiology Laboratories. AiM 3, 504-10 (2013)

20. Vallejo FAG. Epidemiology of Infective Endocarditis Chapter 3 In: Michael S. Firstenberg editor. Contemporary Challenges in Endocarditis. (2016).

21. 21. Baron EJ, Miller JM, Weinstein MP, et al. A Guide to Utilization of the Microbiology Laboratory for Diagnosis of Infectious Diseases: 2013 Recommendations by the Infectious Diseases Society of America (IDSA) and the American Society for Microbiology (ASM) Clin. Infect. Dis. 57, e22-e121 (2013).

22. Lamas C. Diagnostic Strategy for Blood Culture-Negative Endocarditis. Clin. Infect. Dis. 51, 141-2 (2010).

23. Sumatani I, Kagiyama N, Saito C, et al. Infective endocarditis with negative blood culture and negative echocardiographic findings. J. Echocardiogr. 13, 66-8 (2015).

24. Bosshard PP, Kronenbergm A, Zbinden R, et al. Etiologic diagnosis of infective endocarditis by broad-range polymerase chain reaction: a 3-year experience. Clin. Infect. Dis. 37, 167-72 (2001).

25. Lang S, Watkin RW, Lambert PA, Littler WA, Elliott TS. 2004. Detection of bacterial DNA in cardiac vegetations by PCR after the completion of antimicrobial treatment for endocarditis. Clin. Microbiol. Infect. 10, 579-81 (2004).

26. Greub G, Lepidi H, Rovery C, et al. Diagnosis of infectious endocarditis in patients undergoing valve surgery. Am. J. Med. $118,230-8$ (2005). 